\title{
Erfahrungen bei einer Marktrecherche in Vietnam
}

\author{
Sandra Kühn
}

\section{Vorstellung des Unternehmens DRE/CON Großwälzlager GmbH}

Die Firma DRE/CON Großwälzlager GmbH Eberswalde ist eines der mittelständischen Unternehmen, welches den Mut besitzt, den großen Schritt nach Asien zu wagen. Als sogenannter „Zentraler Fertiger deckte der ehemals eigenständiger Bereich der Kranbau Eberswalde $\mathrm{GmbH}$, zugehörig dem damaligen Kombinat TAKRAF, den gesamte Bedarf an Drehverbindungen (DV) und Seilrollen (SR) auf dem Gebiet der neuen Bundesländer ab. Im Jahre 1962 entstand die Fertigungsstätte zur serienmäßigen Produktion von zweireihigen Kugeldrehverbindungen in Eberswalde.

Im Kranbau Eberswalde wurde die Fertigung für Großwälzlager (GWL) eingerichtet, die zentral für alle Kunden in der ehemaligen DDR produzierte. Das Fertigungsvolumen betrug etwa 4.500 Stück pro Jahr. Seit Aufnahme der GWL-Fertigung wurden bis zum gegenwärtigen Zeitpunkt mehr als 95.000 GWL ausgeliefert. Der Umsatz hat sich in den letzten Jahren so entwickelt, daß mittlerweile 75 Mitarbeiter beschäftigt sind. Modernste technische Einrichtungen - CNC gesteuerte Produktionsanlagen, leistungsfähige Hard- und Software - und 35 Jahre Erfahrung als größter Zulieferer der Fördertechnik in Deutschland sind die Gewähr für technisches Know-how und erstklassigen Qualitätsstandard.

\section{Beschreibung der Aufgabenstellung}

Mein Diplomthema lautete: „Erschließung des vietnamesischen Marktes für ein mittelständisches Unternehmen - DRE/CON Großwälzlager GmbH in Eberswalde“. Diese Arbeit enthält eine Zusammenstellung der aktuellen Wirtschaftsdaten zur Erschließung des vietnamesischen Marktes. Die Diplomarbeit ist speziell für das Unternehmen DRE/CON in Eberswalde geschrieben, welches unter anderem Großwälzlager herstellt, die man z. B. in Kränen finden kann. Bei der Bearbeitung wurde auch auf die Einsatzgebiete der Wälzlager eingegangen, da sich hier der Ansatzpunkt für den Export ergibt.

Das Ziel war es, dem Anwender ein Instrument in die Hand zu geben, das ihm einen Überblick über die Absatzchancen eines bestimmten Produkts auf dem vietnamesischen Markt, über mögliche Probleme (z. B. Zollbestimmungen) und über den Umgang mit vietnamesischen Geschäftspartnern verschafft.

\section{Herangehensweise}

Um dem Leser die Aufgabenstellung zu verdeutlichen, habe ich in meiner Diplomarbeit das Unternehmen vorgestellt und die Produkte beschrieben. Wenn man diese Produkte auf dem Exportmarkt vertreiben möchte, muß man wissen, wo diese verwendet werden. Darauf wurde besonders eingegangen, weil das auch gleichzeitig der Ansatzpunkt ist, Großwälzlager in Vietnam zu verkaufen. Um eine Marktrecherche durchführen $\mathrm{zu}$ können, muß man die Produkte des Unternehmens kennen. Hierzu absolvierte ich ein vierwöchiges Praktikum in der DRE/CON Großwälzlager GmbH.

Nachdem die Wirtschaftszweige in Vietnam herausgearbeitet wurden, die diese Produkte benötigen, brauchte man genauere Informationen uiber diese Wirtschaftszweige. Die besten Informationen habe ich aus der Zeitschrift „Asien spezial” und aus dem Internet recherchiert. Es ist beachtlich, wie viele gute Unterlagen man dort bekommt, wenn man viel Zeit mitbringt und die englische Sprache beherrscht.

Nach der Auswertung der Unterlagen trat ich meine Reise nach Vietnam an. Der Flug wurde von dem Unternehmen finanziert, alle anderen anfallenden Kosten mußte ich selber tragen. Ich besuchte Unternehmen (überwiegend in Hanoi), um die Produkte des Unternehmens DRE/CON vorzustellen. Die Unternehmen zeigten großes Interesse, als ich sagte, daß die DRE/CON noch keine Niederlassung in Vietnam hat. Ich lernte sehr schnell, daß vietnamesische Unternehmen sehr gern deutsche Produkte kaufen, auch dann noch, wenn diese $10 \%$ teurer sind als $\mathrm{z}$. B. die dort weit verbreiteten japanischen Güter. „Deutsche Güter haben eine besonders gute Qualität“ - habe ich dort sehr oft gehört!!!

\section{Beschreibung des vietnamesischen Marktes}

Dank der im Jahre 1986 gestarteten und unter dem Namen „Doi Moi“ bekanntgewordenen Politik der wirtschaftlichen Reformen und der schrittweisen Liberalisierung des Außenhandels, hat sich Vietnam in den zurückliegenden Jahren für ausländische Firmen zusehends zu einem attraktiven und zukunftsträchtigen Markt entwikkelt. Das Land, das früher wegen der besonderen politischen Beziehungen zur ehemaligen UdSSR im wesentlichen Kompensationsgeschäfte mit Staatshandelsländern durchführte, wickelt heute etwa $90 \%$ seines Außenhandels mit asiatischen und westlichen Ländern ab. Nach Jahren des Krieges und der politischen Isolierung setzt die Führung in Hanoi eindeutig auf die Kräfte des freien Marktes und unternimmt nachhaltige Schritte zur 
Förderung der Privatinitiative. Im Zuge der Dezentralisierung des Wirtschaftslebens haben die einzelnen Betriebe grundsätzlich das Recht, ihre unternehmerischen Entscheidungen eigenständig zu treffen und entsprechende Verträge abzuschließen.

Nach der schrittweisen Einführung marktwirtschaftlicher Methoden und der Liberalisierung des Außenhandels durchläuft Vietnam einen wirtschaftlichen und gesellschaftlichen Transformationsprozeß, der auch im täglichen Leben nicht zu übersehen ist.

Waren noch vor wenigen Jahren die Straßen von Ho-ChiMinh-Stadt (Saigon) und Hanoi mit Fahrrädern überfüllt, deren Fahrer vornehmlich dunkelgrüne, graue und schwarze Kleidung trugen, bestimmen heute Motorräder das Straßenbild, die demonstrativ und mit viel Lärm abendlich durch die vietnamesischen Metropolen „donnern" und das neue Lebensgefuihl der Jugend symbolisieren. Die zunehmend freundlicheren Farbtöne im Straßenbild beziehen sich keineswegs nur auf die leuchtenden Reklametafeln an den wichtigen Straßenkreuzungen. Auch modische und ausgefallene Kleidung erfreut sich wachsender Beliebtheit. Die Zahl der Pkw's steigt spürbar, und die Nachfrage nach langlebigen Konsumgütern (z. B. elektrische Haushaltsgeräte) zeigt einen deutlichen Anstieg. Inzwischen besitzt in Ho-Chi-MinhStadt mehr als die Hälfte der privaten Haushalte Fernsehgeräte und Motorräder. Auffällig ist ferner, daß zwischen den Händlern derselben Produktgruppe, die gewöhnlich in ein und demselben Stadtteil operieren, keine sichtbare Konkurrenz existiert. Sie verkaufen ihre Produkte wie z. B. Taschen, Schuhe, Kinderkleidung oder Hüte immer in verschiedenen Straßen, aber Stand an Stand. Alle Händler haben die gleichen Produkte. Die Straßen werden auch nach den Gïtern genannt, welche in ihnen verkauft werden, also Hutstraße, Schuhstraße etc. Die Preise sind in der Regel einheitlich und wenig flexibel.

\section{Erfahrungen bei der Verkaufsverhandlung}

Wegen der z. T. sehr unterschiedlichen Lebenseinstellungen und Mentalität hat der deutsche Geschäftsmann beim Umgang mit vietnamesischen Partnern bestimmte Verhaltensregeln zu beachten. Grundsätzlich gilt in Vietnam, wie auch in vielen anderen asiatischen Ländern, daß Arroganz, Überheblichkeit und Besserwisserei nicht geschätzt werden. Gefragt sind vielmehr Höflichkeit, Verständnis und Einfühlungsvermögen in eine andere Denkweise.

Erste Kontakte zu vietnamesischen Geschäftspartnern werden gewöhnlich auf Markterkundungsreisen, Messen oder durch die Vermittlung bestimmter Institutionen, wie die vietnamesische Industrie- und Handelskammer oder von der Technology \& Trade Consulting International (TTCI), hergestellt.

Die Kontaktaufnahme über individuelle Werbeinserate oder Direktanschrift ist ebenso möglich, doch wenig erfolgversprechend. Bei einer schriftlichen Präsentation, die - wenn nicht in Vietnamesisch - in englischer oder französischer Sprache erfolgen sollte, sind in möglichst anschaulicher Form die Tätigkeit des eigenen Unterneh- mens darzustellen und über das angebotene Produkt oder die Dienstleistungen Informationen zu unterbreiten. Zusammen mit einem Schreiben sollten möglichst auch Firmenprospeke (unbedingt in Englisch oder in vietnamesischer Übersetzung) bereitgestellt werden.

\section{1. Über Verhandlungsmentalität Berater zuziehen}

Gesprächstermine sollten vorzugsweise per Brief, Telefax oder Telex angekündigt werden. Telefonische Terminvereinbarungen erscheinen nicht besonders geeignet, da im Vorfeld u. a. sprachliche Verständigungsschwierigkeiten auftreten können.

Termine werden meistens für den Vormittag vereinbart, wobei man damit rechnen muß, ggf. bereits um $8 \mathrm{Uhr}$ einen Termin zu erhalten. Vor solchen Gesprächen erscheint es ratsam, einen vertrauenswürdigen lokalen Berater in die Vorbereitungen mit einzubeziehen. Ein Consultant bzw. erfahrener Landeskenner kann nicht nur interessante Marktinformationen bereitstellen, sondern unter Beriicksichtigung der besonderen Landesmentalität und der Kulturnormen wertvolle Ratschläge für die Verhandlungsführung mit Partnern geben.

Der deutsche Partner hat sich darauf einzustellen, daß er beim ersten persönlichen Kontakt meistens nicht Entscheidungsträgern gegenübersitzt. Insbesondere in staatlichen Stellen ist deswegen oft ein „Nachfassen“ auf höherer Ebene empfehlenswert. Bei vielen größeren Staatsunternehmen ist nicht selten der „zweite Mann“ bzw. Vizedirektor der kompetenteste Gesprächspartner. Während die Direktoren des öfteren aufgrund ihrer politischen Ausrichtung und Zugehörigkeit zur Kommunistischen Partei eine leitende Position halten, verfügen die Stellvertreter über mehr Sachkenntnis und technisches Wissen.

Der deutsche Geschäftsmann sollte auch in Vietnam zu seinen Terminen pünktlich erscheinen. Es ist keine Seltenheit, daß vietnamesische Gesprächspartner zu den Gesprächen bzw. Verhandlungen ohne Jackett (z. T. auch ohne Krawatte) kommen. Bei Verhandlungen auf hoher Ebene sollten jedoch Jacketts bzw. Anzüge getragen werden. Wegen der größtenteils unerfreulichen Erfahrungen aus der Zeit der sozialistischen Planwirtschaft sind Vietnamesen gegenüber Versprechungen grundsätzlich mißtrauisch. Ausländische Partner müssen ihnen genügend Zeit und Gelegenheiten bieten, damit sie zu der Überzeugung gelangen, daß ihr Geschäftspartner vertrauenswürdig und verläßlich ist. Vietnamesen haben eine komplizierte, für westliche Menschen oft schwer verständliche Denkweise und sind gute Beobachter. Emotionale Regungen und Gefühle, die dem unerfahrenen ausländischen Besucher oft unter dem Deckmantel der Höflichkeit und Freundlichkeit verborgen bleiben, prägen nicht selten auch das Verhalten im Geschäftsleben. Es ist u. a. stets darauf zu achten, daß der vietnamesische Partner nicht den Eindruck erhält, „sein Gesicht zu verlieren“. Ein Vietnamese, der vor seinen Mitarbeitern oder Geschäftsfreunden bloßgestellt wird, muß für alle Zeiten mit dem Verlust seiner Autorität rechnen, was tiefgreifende persönliche und berufliche Konsequenzen haben kann. 


\section{Mit Geduld Präsentation abwarten}

Eine der Grundtugenden beim Umgang mit vietnamesischen Geschäftspartnern ist Geduld, die viele Probleme löst. Ferner erleichtern eine positive Grundeinstellung sowie die Bereitschaft, die vietnamesische Kultur, Gesellschaft und Sprache zu verstehen, die Geschäftsbeziehungen.

Die Verhandlungen beginnen i. d. R. in einer formellen Atmosphäre. Detailliertere Fragen werden später erörtert. Gespräche, vor allem in der ersten Phase, werden häufig nicht in Dialogform, sondern als Präsentation geführt, bei denen der Vortragende nach Möglichkeit nicht unterbrochen werden sollte. Gewöhnlich beginnt die vietnamesische Seite mit dem Gespräch, indem ein einführender Vortrag über die eigene Tätigkeit erfolgt. Vietnamesen sind allgemein gute Redner, die die gesamte zur Verfügung stehende Zeit ausschöpfen, um sich zu präsentieren.

Nach der einführenden Darstellung durch die vietnamesische Seite sollte der deutsche Partner in knapper und anschaulicher Form ein Bild von seinem Unternehmen vermitteln. Dabei sollten mehrere anstehende Fragen zugleich vorgebracht werden. Entsprechende Antworten zu den einzelnen Fragen erfolgen meist später. Steht der ausländische Besucher unter dem Eindruck, daß der vietnamesische Partner bestimmten Fragen ausweicht, so kann dies mehrere Gründe haben: Der vietnamesische Partner nicht die geeignete Person, die gestellten Fragen zu beantworten, er hat keine Antworten, er wurde nach vertraulichen Informationen gefragt.

\section{3. „Positives“ hervorheben, Einladungen aussprechen}

Grundsätzlich ist zu beachten, daß in der vietnamesischen Sprache Antworten stets im positiven Sinne formuliert werden; die Benutzung von negativen Wörtern ist nicht üblich. Wenn Vietnamesen „It's no problem“ sagen, meinen sie in Wirklichkeit sehr oft „No, it's a problem". Um eine negative Antwort auch als solche identifizieren zu können, bedarf es somit einer besonderen bzw. umgekehrten Fragestellung, um eventuelle Mißverständnisse zu vermeiden. Darüber hinaus wird des öfteren beobachtet, daß Vietnamesen Versprechungen machen oder gar leichtfertig Verpflichtungen eingehen, ohne alle Auswirkungen ihrer Zusagen zu erkennen und die Komplexität eines Sachverhaltes zu durchblicken.

Bei den Gesprächen sitzen sich die Hauptpersonen beider Seiten an einem Konferenztisch direkt gegenüber. Weitere Personen, die den Verhandlungen beiwohnen, nehmen jeweils rechts und links der Hauptpersonen ihren Platz ein. Auch bei ungünstigem Gesprächsablauf sollte der ausländische Besucher negative Bemerkungen und Reaktionen unterlassen. Von besonderer Bedeutung ist bei Verhandlungen die Rolle des Dolmetschers. Es erscheint empfehlenswert, zu den Gesprächen einen Dolmetscher heranzuziehen, der einem schon vertraut ist und mit dem man bereits früher gut zusammengearbeitet hat.
Ferner ist es ratsam, während der Gespräche Notizen zu machen, da dies großes Interesse signalisiert und die Gegenseite geneigt macht, detailliertere Informationen zu anstehenden Projekten zu unterbreiten.

Es ist üblich, beim ersten persönlichen Treffen Visitenkarten auszutauschen. Ferner sollte der Besucher in der Lage sein, einige Prospekte und Muster seiner Produkte vorzulegen. Die Begrüßung erfolgt i. d. R. durch Händeschütteln. Kleinere Werbe- oder Gastgeschenke (z. B. Kalender, Whiskey) werden gerne angenommen. Auch Humor ist gefragt, solange keine persönlichen Beleidigungen eintreten.

Die Höflichkeit gebietet es, die vietnamesischen Geschäftspartner zu einem Lunch oder Dinner einzuladen. Die Annahme solcher Einladungen kann allgemein als ein günstiges Zeichen für die zukünftige Zusammenarbeit gewertet werden. Es kann davon ausgegangen werden, daß auch der vietnamesische Partner entsprechende Einladungen ausspricht. Solche Zusammenkünfte finden gewöhnlich ohne Ehepartner statt. Die Abendessen dauern i. d. R. nicht sehr lange. Als Zeitdauer für ein Dinner können zwei Stunden angesetzt werden (vorzugsweise zwischen 19 und 21 Uhr).

\subsection{Faktoren, die beim Kauf entscheiden können}

Wenn auch Preise, technische Details sowie Fragen des After-Sales-Service (Reparatur, Wartung, Ersatzteilversorgung, Garantien) bei den Verkaufsverhandlungen eine wichtige Rolle spielen, konzentrieren sich die Schwierigkeiten meist auf Finanzierungsprobleme, da sich die Möglichkeiten der vietnamesischen Seite oft in engen Grenzen halten. Viele vietnamesische Firmenvertreter haben die Einstellung, daß derjenige, der etwas verkaufen will, auch für die Finanzierung zu sorgen hat. Auch wenn die technischen Vorteile eines Produktes gegenüber Konkurrenzangeboten nicht oft genug betont werden können, ist die Unterbreitung günstiger Finanzierungspakete in vielen Fällen ein weitaus wirksameres Instrument der Entscheidungsbeeinflussung im Sinne des Lieferanten.

Einige deutsche Lieferanten weisen bei Verkaufsverhandlungen mit vietnamesischen Firmen darauf hin, daß der angebotene Maschinentyp (z. B. bestimmte Textilund Konfektionsmaschinen) in der VR China (noch) nicht existiert, was offensichtlich stark beeindruckt und als Zeichen hoher Technologie gewertet wird. Deutsche Maschinen erfreuen sich allgemein einer hohen Wertschätzung.

Außerdem erhöhen diverse andere Unterstützungsmaßnahmen des ausländischen Partners die Qualität der Kooperation. So können sich z. B. die Ausbildung von vietnamesischen Mitarbeitern in deutschen Betrieben, Unterstuitzung bei Messebeteiligungen und andere Werbehilfen als nützliche Maßnahmen zur Intensivierung der Zusammenarbeit erweisen.

\section{Schlußbemerkung}

Vietnam hat sich in den vergangenen Jahren wirtschaftlich sehr schnell entwickelt, wenn man bedenkt, daß 
1979 noch ein Krieg gegen die Chinesen stattfand. Trotz der schnellen Entwicklung ist in Vietnam noch sehr viel zu tun: angefangen mit der Verbesserung und dem Bau von Straßen über die Restaurierung von Gebäuden bis hin zur Stabilisierung der Stromversorgung.

Vietnam ist ein großer Absatzmarkt für Großwälzlager (GWL), die z. B. in Kränen oder Baggern zu finden sind. Diese werden überall dort gebraucht, wo gebaut wird - es scheint fast alles modernisierungsbedürftig.

Trotz des hohen Bedarfs an GWL ist es für ein deutsches mittelständisches Unternehmen sehr schwierig, ohne vietnamesische Unterstützung Käufer zu finden. Das sollte aber nicht abschrecken! Man darf nicht noch mehr Zeit verlieren, diesen gewinnbringenden Schritt in den neuen Markt zu wagen. Es ist schon viel zu viel Zeit verstrichen. Wenn man noch länger wartet, ist der Markt dort genauso gesättigt wie in Deutschland. Andere Länder, z. B. Japan, die USA, aber auch Länder wie Schweden, haben das schon viel früher erkannt und sich bereits einen Marktvorteil in Vietnam gesichert.

Die kostengünstigste und am meisten Erfolg versprechende Alternative für die DRE/CON ist der Einstieg in ein Pooling, der von der TTCl Hanoi Ltd. angeboten wird.

Für eine ständige Marktbeobachtung und gezielte Kundenpflege ist die Einschaltung eines lokalen Vertreters bzw. die Einrichtung eines Repräsentationsbüros in HoChi-Minh-Stadt (Saigon) und/oder Hanoi von großer Bedeutung.

Dieser Firmenpool wird der DRE/CON in Vietnam den Einstieg erleichtern und eine Repräsentanz in Vietnam darstellen. Das Vertretungsbüro mit guten Beziehungen zu maßgeblichen Stellen und Personen kann nicht nur aktuelle Informationen zu Investitionsprojekten und zur Bedarfslage einholen, sondern es können über dieses auch Verhandlungen gefuihrt und Lieferverträge vorbereitet werden.

Eine Einbeziehung guter lokaler Berater in die Vertragsverhandlungen hilft ferner, Schwierigkeiten zu überwinden, die durch unterschiedliche Mentalitäten entstehen und vermeidbare Mißverständnisse verursachen können.

Das Studium an der Technischen Fachhochschule war für mich ein voller Erfolg. Hier wurden mir die Türen zu den asiatischen Märkten durch die Unterstützung der Dozenten geöffnet. Das Studium, das sehr praxisbezogen war, endete mit der Diplomarbeit, die ich hier kurz vorgestellt habe. Rückblickend würde ich mich immer wieder für diese Fachhochschule - auf keinen Fall für eine Universität - und für Internationales Marketing als Spezialisierungsfach entscheiden.

\section{Verfasserin}

\section{Sandra Kühn}

(4. Studienjahr, Betriebswirtschaftslehre)

Technische Fachhochschule Wildau

Bahnhofstraße

15745 Wildau 